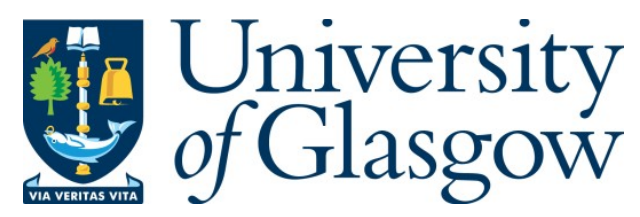

Cobbold, C. A., Lutscher, F., and Sherratt, J. A. (2015) Diffusion-driven instabilities and emerging spatial patterns in patchy landscapes. Ecological Complexity, 24, pp. 69-81.

There may be differences between this version and the published version. You are advised to consult the publisher's version if you wish to cite from it.

http://eprints.gla.ac.uk/111164/

Deposited on: 4 January 2017

Enlighten - Research publications by members of the University of Glasgow http://eprints.gla.ac.uk 


\title{
Diffusion-driven instabilities and emerging spatial patterns in patchy landscapes
}

\author{
Christina A. Cobbold*1, Frithjof Lutscher ${ }^{2}$, Jonathan A. Sherratt ${ }^{3}$
}

September 30, 2015

${ }^{1}$ School of Mathematics and Statistics, University of Glasgow, University Gardens, Glasgow, G12 8QW, United Kingdom.

${ }^{2}$ Department of Mathematics and Statistics, and Department of Biology, University of Ottawa, Ottawa, ON, K1N 6N5, Canada.

${ }^{3}$ Department of Mathematics and Maxwell Institute for Mathematical Sciences, Heriot-Watt University, Edinburgh, EH14 4AS, United Kingdom.

*To whom correspondence should be addressed.

E-mail: christina.cobbold@glasgow.ac.uk

Tel: +44 1413304356

Fax: +441413304111 


\section{Abstract}

Spatial variation in population densities across a landscape is a feature of many ecological systems, from self-organised patterns on mussel beds to spatially restricted insect outbreaks. It occurs as a result of environmental variation in abiotic factors and / or biotic factors structuring the spatial distribution of populations. However the ways in which abiotic and biotic factors interact to determine the existence and nature of spatial patterns in population density remain poorly understood. Here we present a new approach to studying this question by analysing a predator-prey patch-model in a heterogenous landscape. We use analytical and numerical methods originally developed for studying nearest-neighbour (juxtacrine) signalling in epithelia to explore whether and under which conditions patterns emerge. We find that abiotic and biotic factors interact to promote pattern formation. In fact, we find a rich and highly complex array of coexisting stable patterns, located within an enormous number of unstable patterns. Our simulation results indicate that many of the stable patterns have appreciable basins of attraction, making them significant in applications. We are able to identify mechanisms for these patterns based on the classical ideas of long-range inhibition and short-range activation, whereby landscape heterogeneity can modulate the spatial scales at which these processes operate to structure the populations.

\section{Key words}

Diffusion-driven instability, heterogeneous landscape, patch model, pattern formation, predator-prey. 


\section{Introduction}

One of the great challenges in ecology is to uncover and explain the mechanisms that lead to observed spatial patterns of species distributions. For many species, abundance varies spatially as individuals track environmental variation, such as abiotic factors or resources, across a landscape (Leroux et al., 2013; Ergon et al., 2001). Alternatively, spatial distribution patterns can arise in the absence of external forces, due to the pattern-formation mechanism of short-range activation and long-range inhibition (Zelnik et al., in press; Rietkerk et al., 2002; Wang et al., 2010b), or due to density-dependent dispersal leading to phase separation (Liu et al., 2013). These two mechanisms typically create stationary patterns, although moving patterns occur in the presence of advection (Siero et al., 2015; Perumpanani et al., 1995; Sato and Iwasa, 1993). Temporally varying patterns may also arise from asynchronous cycling caused by invasions or obstacles (Sherratt et al., 1995; Petrovskii and Malchow, 2001; Sherratt et al., 2002). The best-studied of these processes is the Turing mechanism, and ecologists have recently identified appropriate long-range inhibition in a number of natural ecosystems and documented corresponding patterns (Rietkerk and van de Koppel, 2008; Deblauwe et al., 2008; Meron, 2012). Our work is concerned with the interplay between extrinsic and intrinsic generation of temporally constant spatial patterns. We develop a theoretical framework and illustrate it with some examples of how environmental variation and intrinsic interaction can combine to create patterns at various spatial scales.

Spatial variation in environmental conditions occurs at various (landscape) scales both naturally, e.g. altitude variation within mountainous regions, and through human intervention, e.g. networks of marine reserves, managed forests, or agricultural systems. Spatial scales of population patterns arising from species interactions (Turing scale) depend on the range of activation and inhibition, i.e. the strength of these interactions and the relative movement of individuals. On one extreme, if the landscape scale is much smaller than the Turing scale, then one can expect to observe intrinsically generated patterns that extend over large regions in space, potentially with small variations to reflect local conditions. Conversely if the landscape scale is large compared to the Turing scale of species interaction, one expects intrinsically generated patterns that change on the long spatial scale of environmental variation (Voroney et al., 1996).

Several authors have studied Turing pattern formation in heterogeneous landscapes. Benson et al. (1993b) investigated pattern formation with constant kinetic parameters and spatially varying diffusion coefficients, see also (Benson et al., 1993a, 1998). Voroney et al. (1996) studied the interplay of Turing patterns and cyclic dynamics that result from a chemical reaction with an additional immobile but spatially heterogeneous complexing agent. Page et al. (2003) considered the generation of patterns near an interface where kinetic parameters change their values abruptly. Subsequent work included smoothly varying monotone and periodic changes in kinetic parameters (Page et al., 2005), see also Garzón-Alvarado et al. (2012) for more intensive numerical simulations in patchy, 2-dimensional domains. Recently Sheffer et al. (2013) and Yizhaq et al. (2014) investigated the interplay between environmental templates and self-organisation in the formation of patterned vegetation in semi-arid regions. Using both theoretical and empirical approaches, they showed that both mechanisms play significant roles in the pattern formation process, with their relative contributions depending on rainfall levels.

In this work, we take a landscape ecology perspective and subdivide the environment into distinct patches. A patch is defined as an environmentally homogeneous geographic region whose spatial extent is comparable to the species' dispersal scale so that a population can be assumed relatively homogeneous within a patch. Population dynamics on each patch are then coupled 
via migration between patches. Such multi-patch models have a long and distinguished history in spatial and community ecology (see for example (Cantrell et al., 2012) for a discussion). In this framework, we study conditions for spatial patterns to evolve in the interesting range where the landscape scale is comparable to the Turing scale (see above). We implement habitat heterogeneity through patch attributes and movement bias.

A series of papers explores pattern formation in epithelia where cell-cell interaction is dominated by nearest-neighbour (juxtacrine) signalling (Owen and Sherratt, 1998; Owen et al., 2000; Webb and Owen, 2004a; O'Dea and King, 2011, 2013; Wearing et al., 2000; Wearing and Sherratt, 2001). In these works, all cells have equal properties (i.e. there is no spatial variation), and interaction between neighbouring cells is non-linear. We will adapt some of the analytical methods used there for our model. A closely related model for a linear inhomogeneous array of coupled chemical reactors was studied in Horsthemke and Moore (2004) as a discretised version of the work in Voroney et al. (1996).

We begin by deriving the predator-prey patch model that forms the basis of our study. We explore emergent patterns with a numerical bifurcation analysis when the number of patches is small. We find a large number of patterns, often stably coexisting, and complex bifurcation diagrams. In the second part, we perform a linear stability analysis when the number of patches is large. For reference and comparison, we identify the stability conditions for the spatially homogeneous model. We compare and contrast these results and discuss the ecological implications of our findings.

\section{The Model}

In a linear landscape of patches of two types (type 1 and type 2), arranged to be periodically alternating, we denote by $u_{1,2}, v_{1,2}$ the respective densities of two interacting species. In our explicit calculations, we focus on predator-prey interaction where a type- 1 patch is suitable for the prey and a type-2 patch is not. Viewing landscapes as mosaics of patches of different quality is common in landscape ecology and also arises in managed ecosystems, for example, a series of marine reserves along a coastline (Botsford et al., 2001; Gouhier et al., 2010) or intercropping in agriculture (Jones and Sieving, 2006).

On a patch of type $i$, the dynamics of these species evolve according to the equations

$$
\dot{u}_{i}=f_{i}\left(u_{i}, v_{i}\right), \quad \dot{v}_{i}=g_{i}\left(u_{i}, v_{i}\right) .
$$

Throughout, we assume that functions $f_{i}, g_{i}$ are sufficiently smooth and that the system preserves non-negativity of solutions.

We denote by $L_{i}$ the length of patch type $i$, and by $L=L_{1}+L_{2}$ and $l=L_{1} / L_{2}$ the landscape period and patch size ratio, respectively. We say that a tile consists of a patch of type 1 and its adjacent patch of type 2 on the right. Hence, a tile represents one period of the landscape (see Figure 1(a)). We denote species' densities on tile $j$ by $u_{1,2}^{j}, v_{1,2}^{j}$. We note here that "tile" is introduced only as a convenient way to describe the system, not as an ecological unit.

We model movement by a discrete diffusion process, so that moving from one good patch to the next requires moving through a bad patch. Individuals of species $u(v)$ leave a patch of type 1 with migration rate $\mu_{u}\left(\mu_{v}\right)$ and move to one of the adjacent patches of type 2 with equal probability. The leaving rate for patch type 2 is multiplied by $\kappa_{u}\left(\kappa_{v}\right)$ to account for patch-dependent dispersal behavior. If $\kappa_{u, v}>1\left(\kappa_{u, v}<1\right)$ then the average time spent in a 
Tile $\mathrm{j}$

(a)

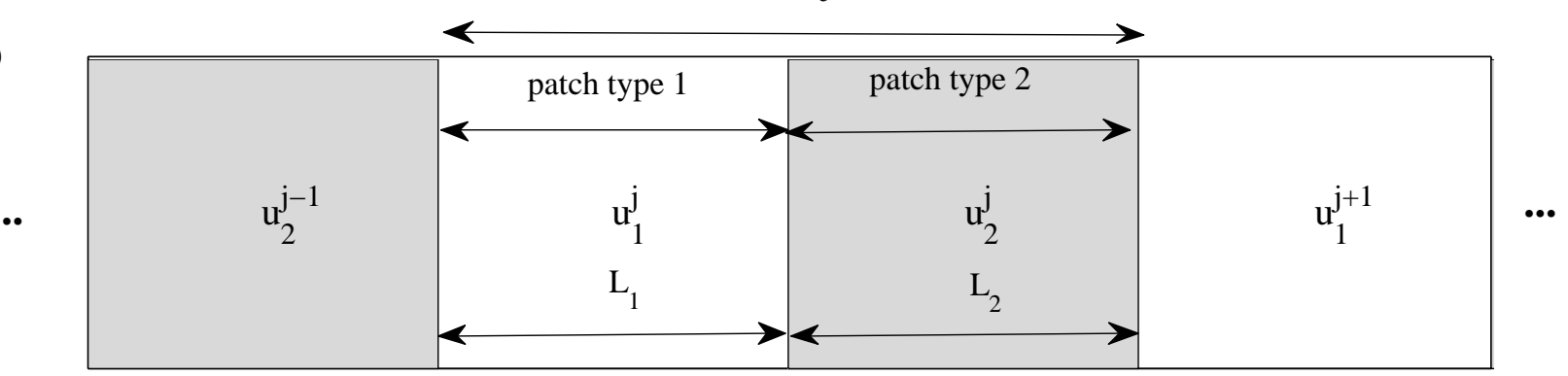

(b)

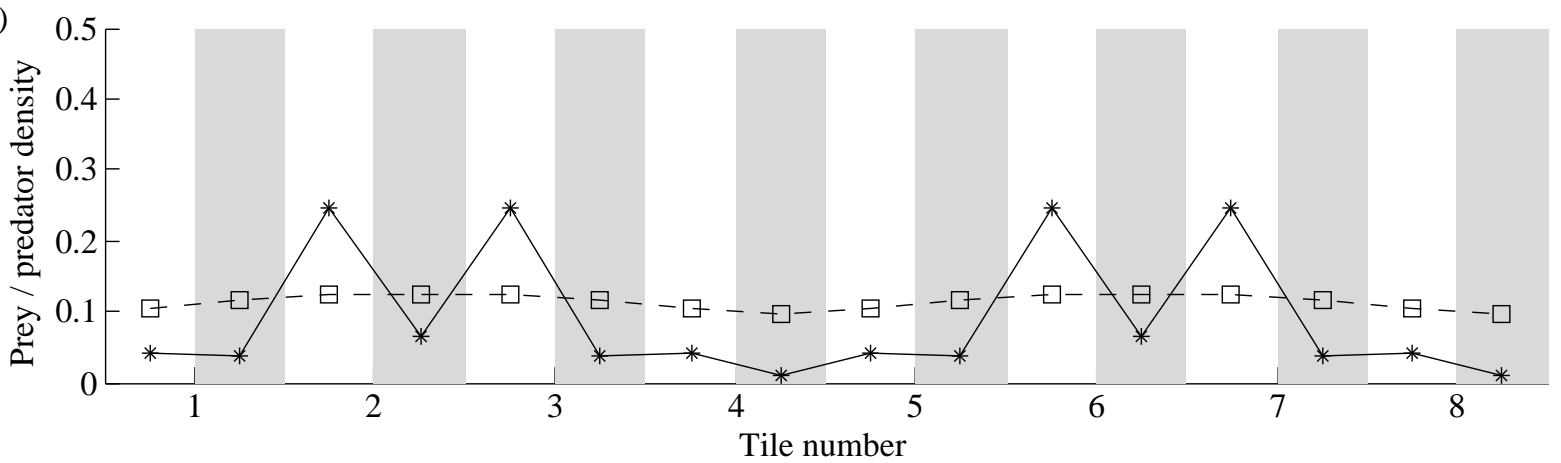

(c)

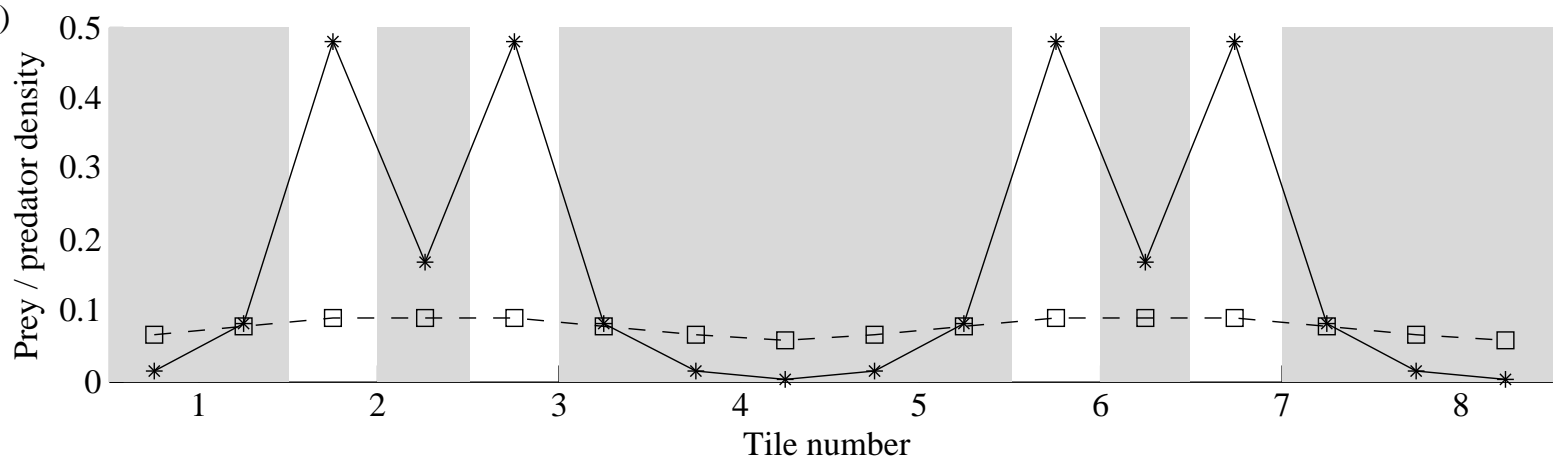

Figure 1: Diagram of patch and tile structure (a) and example pattern solutions (b), (c). (a) illustrates the landscape made up of a series of tiles, with each tile made up of two patches, one of type 1 and one of type 2 with patch sizes $L_{1}$ and $L_{2}$ respectively. (b) illustrates a stationary solution of the model $(2,3)$ for the parameter values $\mu_{u}=0.5, \mu_{v}=5, l=1, b=0.1, s=0.2$, $m=0.6, \kappa_{u, v}=1, q=2.8$. Prey density is denoted by stars and solid lines and predator density by squares and dashed lines. The pattern in prey density $u_{i}^{j}$ is of period 4 on a periodic landscape consisting of 8 tiles. The white regions correspond to the type 1 ('good') patches and the light grey regions correspond to the type 2 ('bad') patches. The prey density in the 'good' patches on tiles $1,4,5$, and 8 is low, in particular it is lower than the prey density in the 'bad' patch on tiles 2 and 6 . (c) illustrates the result of converting the patches 1, 4, 5 and 8 from (b) to bad patches. The result is that the prey density on the remaining good patches is increased while the predator density is decreased on all patches. 
patch of type 2 is shorter (longer), so that overall movement is biased towards patch type 1 (type 2). The spatially coupled model system reads

$$
\begin{aligned}
& \dot{u}_{1}^{j}(t)=\mu_{u}\left[\kappa_{u} \frac{u_{2}^{j}+u_{2}^{j-1}}{2}-u_{1}^{j}\right]+f_{1}\left(u_{1}^{j}, v_{1}^{j}\right), \\
& \dot{u}_{2}^{j}(t)=\mu_{u} l\left[\frac{u_{1}^{j}+u_{1}^{j+1}}{2}-\kappa_{u} u_{2}^{j}\right]+f_{2}\left(u_{2}^{j}, v_{2}^{j}\right), \\
& \dot{v}_{1}^{j}(t)=\mu_{v}\left[\kappa_{v} \frac{v_{2}^{j}+v_{2}^{j-1}}{2}-v_{1}^{j}\right]+g_{1}\left(u_{1}^{j}, v_{1}^{j}\right), \\
& \dot{v}_{2}^{j}(t)=\mu_{v} l\left[\frac{v_{1}^{j}+v_{1}^{j+1}}{2}-\kappa_{v} v_{2}^{j}\right]+g_{2}\left(u_{2}^{j}, v_{2}^{j}\right),
\end{aligned}
$$

where the multiplication of $\mu_{u}, \mu_{v}$ by $l$ in the equations on type-2-patches is the scaling factor that accounts for conservation of individuals. In the case of a finite number of tiles $(N)$ we close the system by assuming periodic boundary conditions such that $u_{i}^{1}=u_{i}^{N}$ and $v_{i}^{1}=v_{i}^{N}$. Periodic boundary conditions allow for easy comparison to dynamics on an infinite domain, moreover they are equivalent to Neumann boundary conditions on a domain of length $N / 2$.

\section{Dynamics on a patch}

On patches of type 1 ('good') we choose the non-dimensional Leslie or May model (May, 1974; Strohm and Tyson, 2009; Mukhopadhyay and Bhattacharyya, 2006) for predator species $v$ and prey species $u$, given by

$$
f_{1}(u, v)=u(1-u)-\frac{u v}{b+u}, \quad g_{1}(u, v)=s v\left(1-\frac{v}{q u}\right) .
$$

In this scaling, $b$ denotes the half-saturation constant of the Holling type II functional response. The predator grows logistically with intrinsic rate $s$ and carrying capacity $q u$. This formulation arises from the assumption of variable predator-territory size (Turchin, 2001).

Patches of type 2 ('bad') are unsuitable for the prey so that we replace the logistic growth term by a linear death term. Predator dynamics depend only on prey abundance and not on patch type. Hence, model equations on patches of type 2 are given by

$$
f_{2}(u, v)=-m u-\frac{u v}{b+u}, \quad g_{2}=g_{1} .
$$

On an isolated good patch, there is a unique positive steady state, given by

$$
u_{*}=\frac{1}{2}\left(1-b-q+\sqrt{(1-b-q)^{2}+4 b}\right), \quad v_{*}=q u_{*} .
$$

Parameter $q$ is the ratio of predator-to-prey steady-state densities and will be used as a bifurcation parameter later. The community matrix at this state,

$$
J=\left[\begin{array}{cc}
1-2 u_{*}-\frac{b\left(1-u_{*}\right)}{b+u_{*}} & -\frac{u_{*}}{b+u_{*}} \\
s q & -s
\end{array}\right],
$$

has positive determinant. The stability therefore depends on the sign of the trace. The trace is zero when

$$
1-2 u_{*}-b \frac{1-u_{*}}{b+u_{*}}=s
$$


If $s$ is large, then this equation has no solution and the steady state is stable. If $s$ is small enough, there are two critical values $q_{H, 1}<q_{H, 2}$ where a Hopf bifurcation occurs. The steady state is unstable for $q_{H, 1}<q<q_{H, 2}$ and a stable limit cycle exists. Depending on parameter values, the bifurcation at $q_{H, 2}$ may be subcritical so that a limit cycle may exist for values $q>q_{H, 2}$ (Gasull et al., 1997). For the parameter values we use in the next section $(b=0.1, s=0.2)$, these critical points are $q_{H, 1} \approx 0.895$, and $q_{H, 2} \approx 4.05$, and the latter bifurcation is subcritical.

\section{Dynamics on a tile}

When we couple the dynamics on a good patch with those on a bad patch, migration has a stabilising effect on the dynamics. For all parameters sets that we have studied, numerical investigation suggests that there is a unique positive stable coexistence steady state. We do not attempt to find exact conditions for when this happens since our focus is on the question of spatial pattern formation at a landscape level.

Qualitatively, this stabilisation occurs when the bad patch is large enough, movement rates are large enough, and movement preference for the good patch is not too strong. The periodic orbits for intermediate values of $q$ on a single good patch can also be present on a tile if the influence of the bad patch is weak enough. The latter scenario arises, for example, when the size of the good patch is much larger than that of the bad patch, when migration rates are very small so that the patches are only weakly coupled, or when migration preference for the good patch is particularly strong.

For our base-line parameters, we fix patch sizes to be equal $(l=1)$ and choose migration without patch preference $\left(\kappa_{u, v}=1\right)$. We also fix migration rates so that the prey moves much less $\left(\mu_{u}=0.5\right)$ than the predator $\left(\mu_{v}=5\right)$. The population dynamics parameters are fixed at $b=0.1, s=0.2$, and $m=0.6$. Then, numerically, the dynamics on an entire tile show a unique, globally stable positive steady state for all $q \in(0,10]$ even though the dynamics on a single good patch can have oscillations for intermediate values of $q$. We will return to some aspects of cyclic dynamics in section 3.2 .

\section{Methods and Results}

We structure our analysis of pattern formation in the heterogeneous landscape into two parts. First we use a numerical bifurcation method to study patterns when the number of tiles is relatively small. Depending on our bifurcation parameter $q$, we document a large number of complex, stable, steady spatial patterns. Secondly, we use linear analysis to derive the dispersion relation of the 'spatially homogeneous steady state' on an infinite patchy landscape. This approach allows us to identify stability boundaries and the onset of spatial patterns with respect to all other parameters, in particular those parameters governing movement and landscape attributes. Finally, we discuss the similarities and differences between the two approaches.

The term 'homogeneous steady state' warrants some explanation. Our system does not support a homogeneous steady state in the classical sense where prey and predator densities are constant in space, i.e. independent of patch type. However, if we consider the tile as the basic spatial unit, we do obtain a steady state solution where each of the four densities $u_{1}^{j}, u_{2}^{j}, v_{1}^{j}$ and $v_{2}^{j}$ is independent of tile-number $j$. We refer to this solution as our homogeneous solution or tile-independent solution.

Unless otherwise stated explicitly, parameter values in this section are $\mu_{u}=0.5, \mu_{v}=5$, $l=1, b=0.1, s=0.2, m=0.6, \kappa_{u, v}=1$ and $q=1.8$. 


\subsection{Numerical bifurcation results for small systems}

The simplest solution of our model is the homogeneous steady state. Our extensive program of numerical simulations suggests that when parameter $q$ is either sufficiently small or sufficiently large, there is a unique solution of this type, which is globally stable.

For intermediate values of $q$, simulations reveal "patterns" by which we mean locally stable time-independent solutions in which the predator and prey densities are not the same in all tiles. We undertook a numerical investigation of such patterns via numerical bifurcation analysis, for which we used the software package AUTO (Doedel, 1981; Doedel et al., 1991, 2006). For a relatively small value of $q$ (e.g. $q=1$ ) we calculated numerically the $j$-independent solution. We then continued this solution numerically, looking for bifurcations to patterned solutions and then continuing these pattern solution branches. AUTO is able to detect not only the existence of patterns, but also to determine their stability as model solutions. This approach to investigating periodic solutions of spatially discrete systems has been used previously in developmental biology, for epithelia in which there is direct cell-cell contact via juxtacrine signalling (Wearing and Sherratt, 2001; Webb and Owen, 2004b; O'Dea and King, 2013). Although it is simple in concept, the approach raises many technical difficulties in the present context, and we discuss these in detail in Appendix A, focussing here on the results of our analysis.

Figure 2 shows the bifurcation diagrams for $N=2,4,6,8$ tiles, plotting the values of $u_{1}^{j}$ against $q$; (examples of bifurcation diagrams for odd number tiles can be found in the supplementary material) . The thin black lines denote unstable patterns (spatially non-constant steady states), and the thin yellow-black dashed lines denote unstable tile-independent solutions. The thick bright yellow lines are stable tile-independent ("period 1") solutions, and the other thick coloured lines denote stable patterns; representative patterns are shown in the same colours above the main plot. The black stars denote results from a series of 1000 simulations for each of $q=1.0,1.25,1.5, \ldots$. Here we solved the equations with initial conditions in which each variable was chosen randomly from a uniform distribution between $20 \%$ and $200 \%$ of its value in the tile-independent solution. In these simulations, we solved for a long time and then plotted the values of $u_{1}^{j}$ for each $j$.

For $N=2$, the bifurcation diagram is relatively simple. The tile-independent solution is stable for $q<1.89$ and $q>4.03$. At these two critical values it changes stability, giving rise to a looped branch of period-2 solutions. For $N=4$, the tile-independent solution loses stability a little earlier, at $q=1.59$, with a patterned solution branch emanating subcritically. There are three different stable portions of patterned solution branches, with small overlaps. These overlaps imply two coexisting stable patterns, and this is confirmed by simulation results for $q=3.75$, with $607 / 1000$ of the initial conditions generating the purple pattern, and the remaining 393/1000 giving the bright green pattern. Note that a doubled version of the pattern solution branch for $N=2$ is necessarily also a solution for $N=4$, but it is unstable on the larger domain. For $N=6$ the number of solution branches is significantly greater, forming a complicated network, and there are eight separate stable sections of solution branches: one of period 2, two of period 3, and five of period 6 . The brown solution branch is a tripled version of the pattern solution branch for $N=2$ : the whole of this branch is necessarily a solution for $N=6$, but only a small part of it is stable. For some values of $q$ there are three coexisting stable patterns, all of which are observed in our simulations. For $N=8$ the bifurcation is slightly simpler, but again there are multiple coexisting stable patterns for significant ranges of $q$.

To illustrate the rapidly increasing complexity of emergent patterns, Figure 3 shows the 

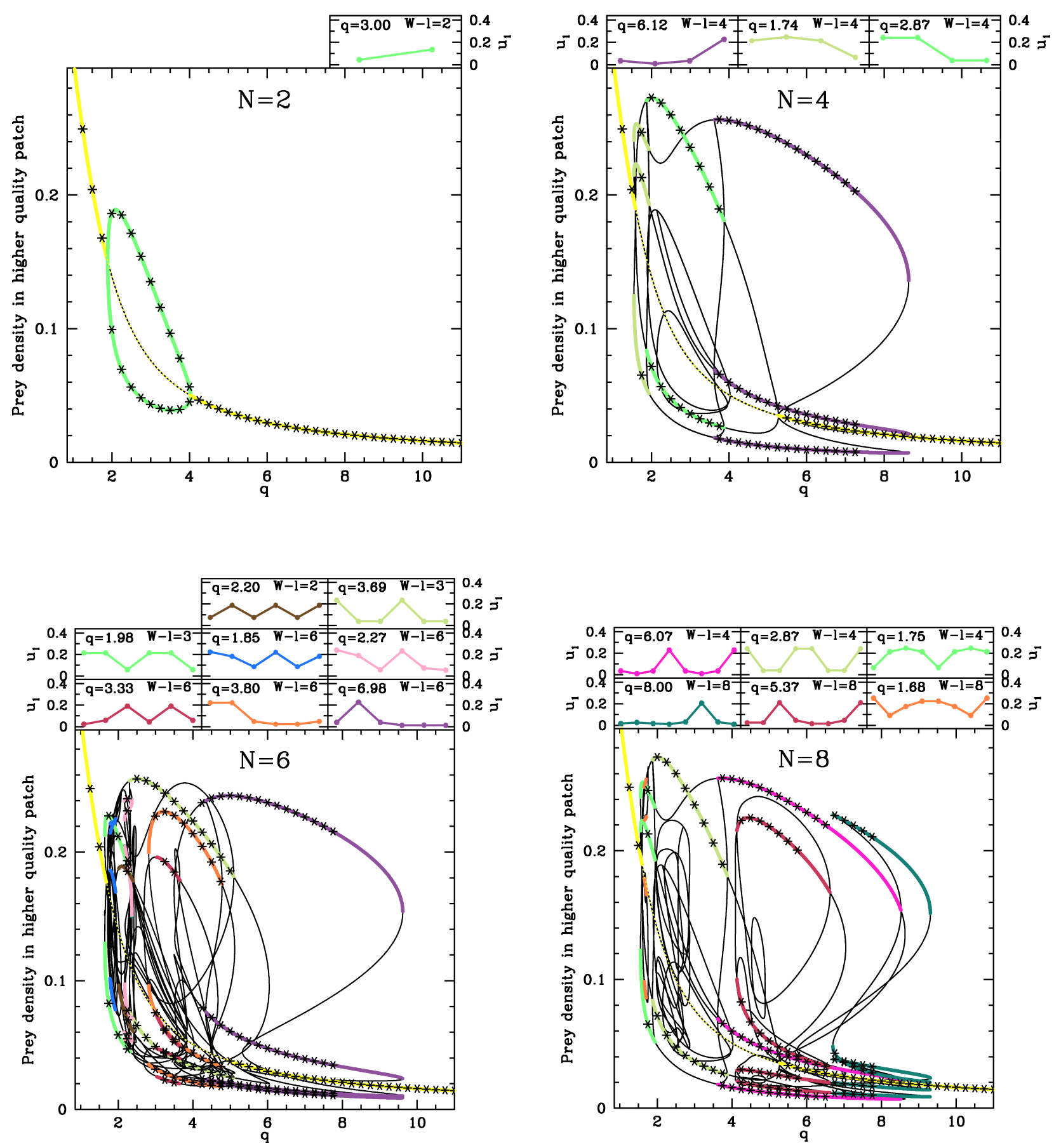

Figure 2: Bifurcation diagrams showing the values of $u_{1}^{j}$ in stationary solutions of the model $(2,3)$ as a function of $q$, for $N=2,4,6,8$ tiles. Thin black lines denote unstable solutions, thick bright yellow lines denote stable tile-independent (period-1) solutions, and thick coloured lines denote patterns. Each stable part of a solution branch is plotted in a different colour, and representative examples of the corresponding patterns are shown above the main figure panels. Black stars denote results from a series of 1000 simulations for each of $q=1.0,1.25,1.5, \ldots$. Here we solved the equations with the value of each variable at $t=0$ chosen randomly from a uniform distribution between $20 \%$ and $200 \%$ of its value in the tile-independent solution. We plot the values of $u_{1}^{j}$ for each $j$ at $t=10^{8}$ : this large solution time is necessary because there can be long transients near unstable solutions. To avoid numerical solutions getting trapped near solutions that are only just unstable, we used a small absolute tolerance of $10^{-8}$. 
results for $N=12$ and $N=16$. The network of solution branches is so complicated that in many places no space is visible between them, and there are many stable pattern branches: 19 for $N=12$ and 54 for $N=16$. Moreover the wide variety of coexisting stable patterns is reflected in the results of our simulations: for most values of $q$ in our range, many different patterns develop, depending on initial conditions. Note that to improve clarity, we do not show simulation results in Figure 3 but they are included in the online supplementary material, where we show bifurcation diagrams for $N=2,3, \ldots, 10,12$ and 16 , plus representative patterns from each stable portion of a solution branch.

The overall message of our results is that unless the number of tiles $N$ is very small, there is a rich and highly complex array of stable patterns, located within an enormous number of unstable patterns. Moreover our many of the stable solution branches arise in out simulations using random initial conditions, which indicates that they have appreciable basins of attraction, and should therefore be observable in real systems. Since the numerical bifurcation methods applied in this section require intensive computations, we present an alternative approach to study pattern formation in the next section.

\subsection{Dispersion relation, stability and patterns}

An analytic approach for studying pattern-formation conditions is to linearise at a spatially constant steady state and to derive the dispersion relation that gives the temporal growth rate of perturbations of a certain wave number. This technique is well established in reactiondiffusion equations (Murray, 2001) and coupled lattices (Webb and Owen, 2004a; Wearing et al., 2000; Lubensky et al., 2011) and networks (Wolfrum, 2012). As discussed previously, we obtain a homogeneous solution only on the level of tiles. We denote this tile-independent state as $\left(u_{1}^{*}, u_{2}^{*}, v_{1}^{*}, v_{2}^{*}\right)$. The spatial relation of $u_{1}^{*}, v_{1}^{*}$ and $u_{2}^{*}, v_{2}^{*}$ within a tile needs to be reflected in the perturbation ansatz. Hence, after we linearise the equations in (2), we look for solutions of the form

$$
\tilde{u}_{1}^{j}(t)=\bar{u}_{1} \exp (\sigma t+j k \mathrm{i}), \quad \tilde{u}_{2}^{j}(t)=\bar{u}_{2} \exp \left(\sigma t+\left(j+\frac{1}{2}\right) k \mathrm{i}\right),
$$

and similarly for $v_{n}$, where $\bar{u}_{n}$ is a constant, and $\mathrm{i}^{2}=-1$. The temporal growth rate of the solution is given by $\sigma$, the wave number is $k$, and $j$ is the discrete (integer) distance corresponding to tile number. To interpret $k$ as a wave number corresponding to wavelength $N$ on the lattice, it needs to be of the form $k=N / 2 \pi$; however, for analytical purposes, it is helpful to consider it as a continuous variable. Here, $N$ is shortest number of tiles needed to see a pattern of that wave length, this is not the same as the $N$ defined in section 3.1, but it is closely related and so we use the same letter. Since the centre of a type-2-patch is halfway between two consecutive type-1-patches (see Figure 1(a)) we need to evaluate the linearisation on bad patches at $j+1 / 2$, as it appears in (8).

The desired solutions exist if the constants $\overline{\mathbf{x}}^{T}=\left(\bar{u}_{1}, \bar{u}_{2}, \bar{v}_{1}, \bar{v}_{2}\right)$ satisfy the linear system $(M-\sigma I) \overline{\mathbf{x}}=\mathbf{0}$, where

$$
M=\left(\begin{array}{cccc}
-\mu_{u}+f_{1 u} & \kappa_{u} \mu_{u} \cos (\pi / N) & f_{1 v} & 0 \\
l \mu_{u} \cos (\pi / N) & -\kappa_{u} l \mu_{u}+f_{2 u} & 0 & f_{2 v} \\
g_{1 u} & 0 & -\mu_{v}+g_{1 v} & \kappa_{v} \mu_{v} \cos (\pi / N) \\
0 & g_{2 u} & l \mu_{v} \cos (\pi / N) & -\kappa_{v} l \mu_{v}+g_{2 v}
\end{array}\right),
$$

and $N=2 \pi / k$ is the wavelength as above. Partial derivatives of the interaction terms are 


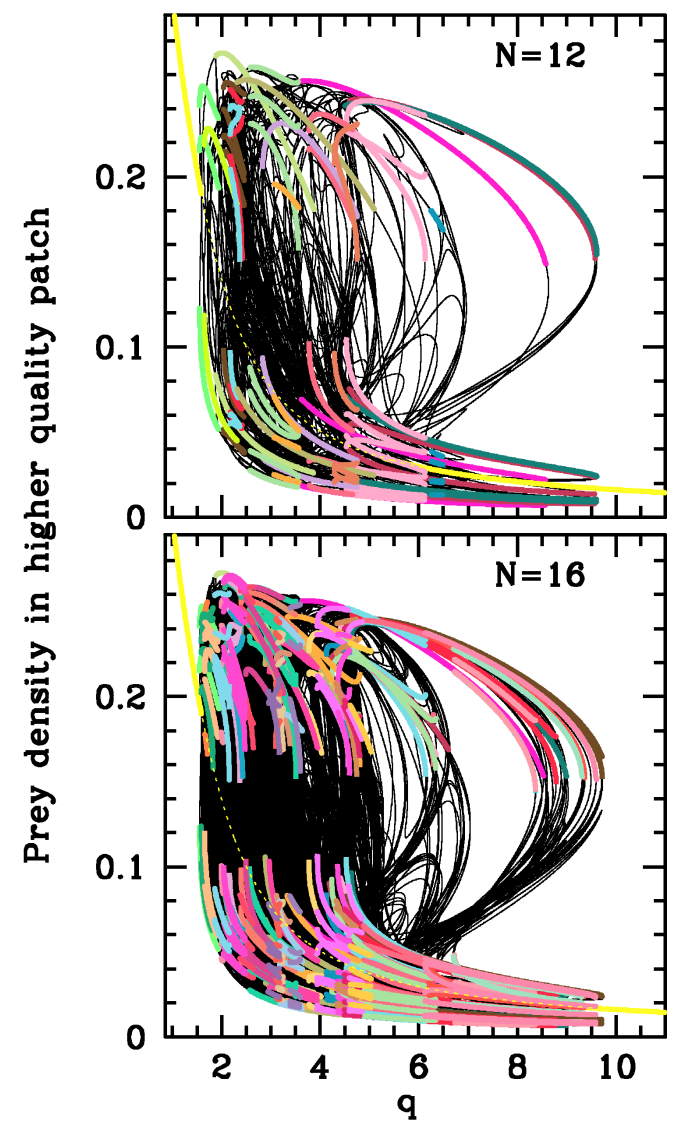

Figure 3: Bifurcation diagrams as in Figure 2 for $N=12$ and 16 tiles. For improved visual clarity we omit simulation results, but otherwise all details are as in Figure 2. In view of the large number of stable portions of solution branches, we do not show examples here, but a representative pattern from each stable portion is plotted in the online supplementary material. 
denoted by subscripts, for example

$$
f_{1 u}=\left.\frac{\partial f_{1}}{\partial u}\right|_{\left(u_{1}^{*}, v_{1}^{*}\right)}, \quad g_{2 v}=\left.\frac{\partial g_{2}}{\partial v}\right|_{\left(u_{2}^{*}, v_{2}^{*}\right)},
$$

and the other terms analogously. From the condition that the solution to this linear system be non-trivial, we obtain the dispersion relation

$$
F(k, \sigma)=\operatorname{det}(M-\sigma I)=0 .
$$

For spatial pattern formation we require the steady state to be (i) stable to homogeneous perturbations (i.e. $\Re(\sigma)<0$ when $k=0$ ), and (ii) unstable to inhomogeneous perturbations (i.e. $\Re(\sigma)>0$ for some $k \neq 0$ ). We illustrate and discuss the stability boundary of the $j$ independent solution in several figures below. In each figure, we indicate whether a perturbation of wavelength $N$ can grow with a positive real eigenvalue $(\sigma>0$, white region) or will decay with a real negative eigenvalue $(\sigma<0$, black region) or non-real eigenvalue with negative real part $(\Re(\sigma)<0$, grey region). We expect patterns to form in the white region. To generate these figures, we calculated the stable tile-independent steady state by numerically solving Equations (2) on a single tile with periodic boundary conditions. For each wavelength, we then found the characteristic polynomial of $M$ and evaluated its roots numerically (using the root command in MATLAB). We focus our results on the effects of movement-related parameters.

\section{Relative dispersal ability}

A key requirement for classical diffusion-driven pattern formation is a difference in dispersal ability, to achieve short-range activation and long-range inhibition (Murray, 2001). Since the prey corresponds to the activator in our model and the predator to the inhibitor, we expect that patterns form when the relative dispersal ability $\mu_{v} / \mu_{u}$ is large enough. Figure 4 (a) shows essentially this behaviour, but the situation is slightly more complex than in the case of a homogeneous landscape. In Figure 4 (a) we fixed $\mu_{u}=0.5$ and varied $\mu_{v}$. When $\mu_{v}=\mu_{u}$, no patterns form. As $\mu_{v}$ increases, patterns of wavelength 3 and 4 emerge, and the range of unstable wavelengths increases as $\mu_{v}$ increases. Note that the white region between $N=1$ and $N=2$ corresponds to non-integer wavelengths and is not observable on our lattice. We note that a different class of spatio- temporal dynamics arises when predator dispersal is very small. The dispersion relation then predicts periodic traveling waves, i.e. instabilities with non-real $\sigma$ and $\Re \sigma>0$. This scenario is present in panel (a) for values of $\mu_{v}$ below 0.1004 (thin white strip at the bottom of the figure). The dynamics on an isolated good patch are oscillatory, and prey dispersal propagates these oscillations in space to generate periodic traveling waves.

In Figure 4 (b) we instead fixed $\mu_{v}=5$ and varied $\mu_{u}$. When $\mu_{u} \geq 1$, no patterns form. As $\mu_{u}$ decreases, patterns with small wavelengths $(3 \leq N \leq 6)$ emerge as expected from the previous scenario. The choice of $\mu_{v}$ seems to constrain the range of unstable wavelengths that can be obtained by varying $\mu_{u}$, but not vice versa (compare panel (a)). Rietkerk and van de Koppel (2008) also observed the key role of long distance negative feedback in determining the existence and regularity of patterns. As $\mu_{u}$ decreases even further, the homogeneous state becomes stable again, even though the ratio $\mu_{v} / \mu_{u}$ is large. In this case, the range of activation becomes too small to spread across the neighbouring bad patch since the residency time in the good patch $\left(\frac{1}{\mu_{u}}\right)$ is high. For the chosen parameter values, the dynamics on an isolated good patch are oscillatory (as discussed in section 2), but the relatively large predator movement stabilises the dynamics. The analysis suggests mobile predators and prey are both needed to observe patterns, however a low predator residency time in good patches appears to be an important ingredient for determining the wavelength of resulting patterns. 


\section{Movement bias $\kappa_{u}$ and $\kappa_{v}$}

The dispersion relation predicts that no patterns form when prey movement is heavily biased towards good patches (e.g. $\kappa_{u}>1.4$ in Figure 4 (c)). As $\kappa_{u}$ decreases, perturbations of relatively small wavelengths ( $2 \leq N \leq 6$ for the chosen parameters) become unstable and patterns arise. Even though the movement rates are constant in this figure, the emergence and disappearance of patterns can be explained in terms of the relative scales of activation and inhibition as follows. By decreasing $\kappa_{u}$, the residence time in bad patches $\left(\frac{1}{\mu_{u} \kappa_{u}}\right)$ is increased, which effectively increases travel time between two consecutive good patches. Thereby the activation range decreases. Vice versa, increasing $\kappa_{u}$ decreases the residence time in bad patches. Effectively, prey move faster through the landscape, thereby increasing the activation range and destroying any potential patterns.

With movement bias of the predator, the same mechanisms are in effect. Since long-range inhibition aides pattern formation, these mechanisms produce contrasting results (not shown). As $\kappa_{v}$ increases, predators bias their movement towards good patches by decreasing their residence time in the bad patches. This behaviour effectively increases their overall movement rate, and with increased inhibition range, patterns may form.

\section{Patch size}

Pattern formation can occur for intermediate size of good patches relative to bad patches. Figure $4(\mathrm{~d})$ shows the case of fixed $L_{2}$ and varying $L_{1}$, but the reverse case is qualitatively the same. When the ratio $l=L_{1} / L_{2}$ is small, prey growth on good patches cannot compensate for prey death in bad patches to produce enough activation for patterns to form. At intermediate ranges, good patches are large enough to enhance prey growth and bad patches are large enough to stabilise the oscillatory dynamics on good patches. When $l$ is large, then the oscillatory dynamics on a good patch cannot be stabilised by the (relatively) small bad patches, and the dynamics on each tile are oscillatory. Due to movement, these local oscillations then form periodic traveling waves. Webb and Owen (2004b) also found periodic travelling waves in their lattice model of intracellular signalling. As the focus of the current work is the study of stable patterns we leave the study of the periodic travelling waves for future work.

\section{Homogeneous versus heterogeneous landscapes}

To complete this section, we ask what effect the bad patches have on the occurrence of patterns compared to a homogeneous landscape. When all patches are good patches (i.e. $f_{1}=f_{2}, g_{1}=g_{2}$ ), then we have a homogeneous landscape, consequently there is no patch preference (i.e. $\kappa_{u, v}=1$ ). In this case, the four-dimensional system (9) reduces to two equations, and the dispersion relation can be written explicitly as

$$
\begin{array}{r}
K^{2}\left(\mu_{u} \mu_{v}\right)(1+l)^{2}+K\left[\sigma\left(\mu_{u}+\mu_{v}\right)(1+l)-a_{11} \mu_{v}(1+l)-a_{22} \mu_{u}(1+l)\right] \\
+\left[\sigma^{2}-\sigma\left(a_{11}+a_{22}\right)+a_{11} a_{22}-a_{12} a_{21}\right]=0
\end{array}
$$

where $K=\sin ^{2}(k / 4)$ and $a_{i j}$ are the entries in the community matrix $J$ given in Equation (6), i.e. $a_{11}=f_{1 u}, a_{22}=g_{1 v}$ and so on. The conditions for diffusion-driven instabilities in this dispersion relation are

$$
\begin{gathered}
a_{11}+a_{22}<0, \quad a_{11} a_{22}-a_{12} a_{21}>0, \quad a_{11} \mu_{v}+a_{22} \mu_{u}>0, \\
4\left(a_{11} a_{22}-a_{12} a_{21}\right) \mu_{u} \mu_{v}<a_{11} \mu_{v}+a_{22} \mu_{u} .
\end{gathered}
$$


(a)

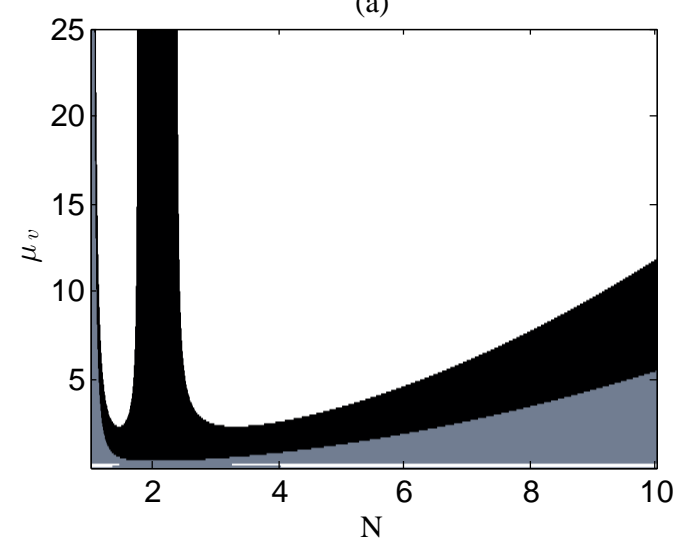

(c)

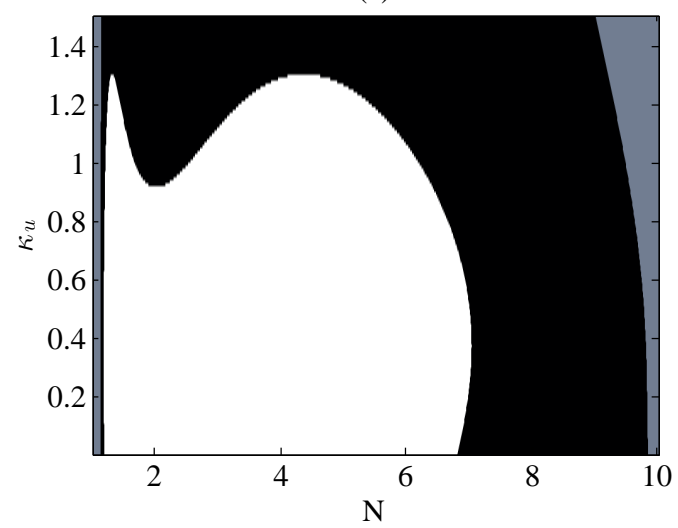

(b)

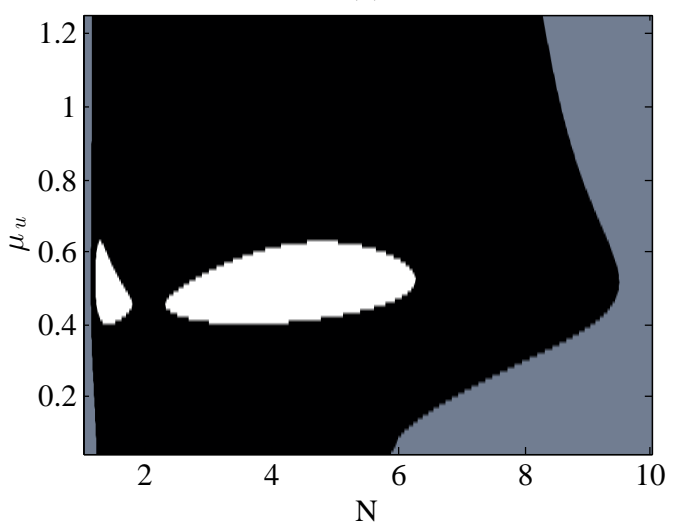

(d)

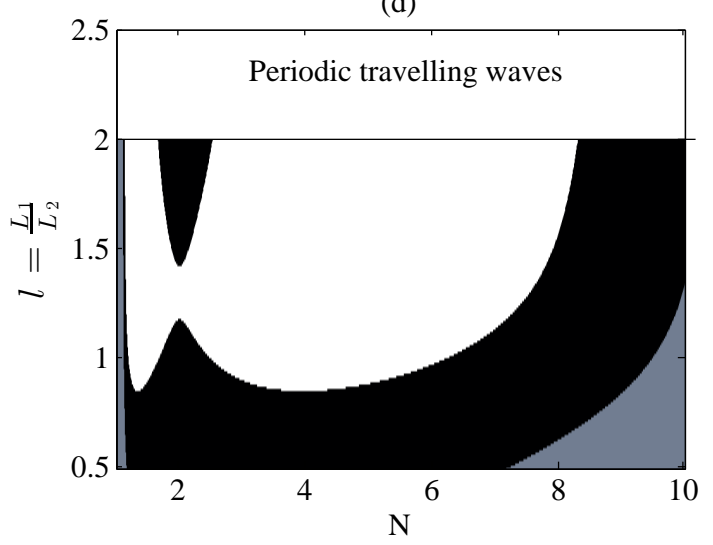

Figure 4: Stability boundaries illustrating the outcome of the linear stability analysis of the patch-independent (period 1) solution on an infinite, one-dimensional spatial domain. The white regions indicate values of the parameter ( $y$-axis) for which we expect to obtain a pattern of wavelength $N$ (x-axis). In the white region the patch-independent solution is stable to spatially homogeneous perturbations, and unstable to spatially varying perturbations of wavelength $N$. In the black and grey regions the period 1 solution is stable to spatially varying perturbations of wavelength $N$. In the black regions, the dominant eigenvalue associated with spatially varying perturbations of wavelength $N$ is real, in the grey regions, it is not. We illustrate in (a) the effect of predator migration rate, (b) the effect of prey migration rate, (c) the effect of prey patch preference, and (d) the effect of relative patch size, on pattern formation. In both (a) and (d) periodic travelling wave solutions are predicted; this occurs in the small white region at the bottom of figure $(\mathrm{a})\left(\mu_{v} \approx 0.1004\right)$ and in the top region of figure $(\mathrm{d})(l \geq 2.0276)$. 
These conditions are the familiar ones for reaction-diffusion equations with movement rates $\mu_{u, v}$ replacing diffusion constants (cf. Murray (2001)). This similarity is understandable since in a homogeneous landscape, our model is essentially a midpoint discretisation of a continuous-space model. Note that relative patch size $l=L_{1} / L_{2}$ drops out from the relation, as it should in a homogeneous landscape.

The two plots in Figure 5 illustrate the difference in the stability behaviour of the tileindependent solution for a homogeneous (two good patches per tile, left plot) and heterogeneous (a good and a bad patch per tile, right plot) landscape. (We use two good patches per tile so that we can compare the length scales of the emergent patterns between the two types of landscapes.) In the homogeneous landscape, only a very narrow range of $q$ leads to pattern formation for a limited range of wavelengths $N$ (white region). There is a large region of oscillatory solutions when $q<4$ (see Section 2), but the entire region $q>5$ has a stable homogeneous solution. In the heterogeneous landscape, the region of pattern formation is much larger (white region, right plot). The presence of bad patches stabilises all the oscillations for $q<4$ so that spatial patterns can emerge there. In addition, patterns can arise for values of $q$ up to at least 7; much larger than in the homogeneous case. We hypothesise that the small-scale variation in the steady-state densities that is generated by the presence of bad patches can act as a catalyst that favours pattern formation.

\subsection{Comparison of the different approaches}

The numerical continuation method in Section 3.1 revealed a great number of coexistent spatial patterns, but was limited to a single bifurcation parameter and required intensive computations. The analytical dispersion-relation method in Section 3.2 captures the stability behaviour of the tile-independent state in an infinite landscape relatively easily, but cannot detect other patterns and is based only on linear stability. We compare the two methods in Figure 6. For each wavelength $(N)$, the hashed bars in (a) indicate the range of $q$ for which the tile- independent solution is unstable according to the numerical method applied to the nonlinear model. The white bars in (a) indicate the values of $q$ for which (locally stable) non-trivial spatial patterns exist. The white region in (b) corresponds to linear instability of the tile-independent state according to the dispersion relation.

We see that the instability region for finitely many tiles (hashed bars, panel (a)) correspond reasonably well to the instability region on the infinite landscape (white region, panel (b)), but that the pattern formation region (white bars, panel (a)) is much larger than the instability region of the tile-independent solution. Specifically, we saw in Figure 2 that all primary bifurcations from the period-1 pattern are sub-critical. Despite this, the linear analysis still predicts the patterns for small wavelengths with reasonable success. For example, in the case $N=4$, a period-4 pattern branches sub-critically from the period-1 pattern, and only becomes stable once it folds back. Secondary bifurcations lead to additional patterns that are stable and fold back to the period-1 pattern long after this period-1 pattern is stable again. As the propensity for secondary bifurcations increases, the ability of the linear analysis to predict patterns decreases. Diffusion-driven instabilities arising in reaction-diffusion models typically result from supercritical solutions so that the linear stability analysis predicts patterns well, at least close to the bifurcation point. In discrete-space systems, however, sub-critical bifurcations are common (O'Dea and King, 2013). And even continuous-space systems can exhibit numerous sub-critical bifurcations in the presence of an advection term (Sherratt, 2013; van der Stelt et al., 2013; Siteur et al., 2014). Hence, the linear stability analysis can serve as an entry point into studying pattern formation, but to obtain the full picture, one has to consider the nonlinear model 

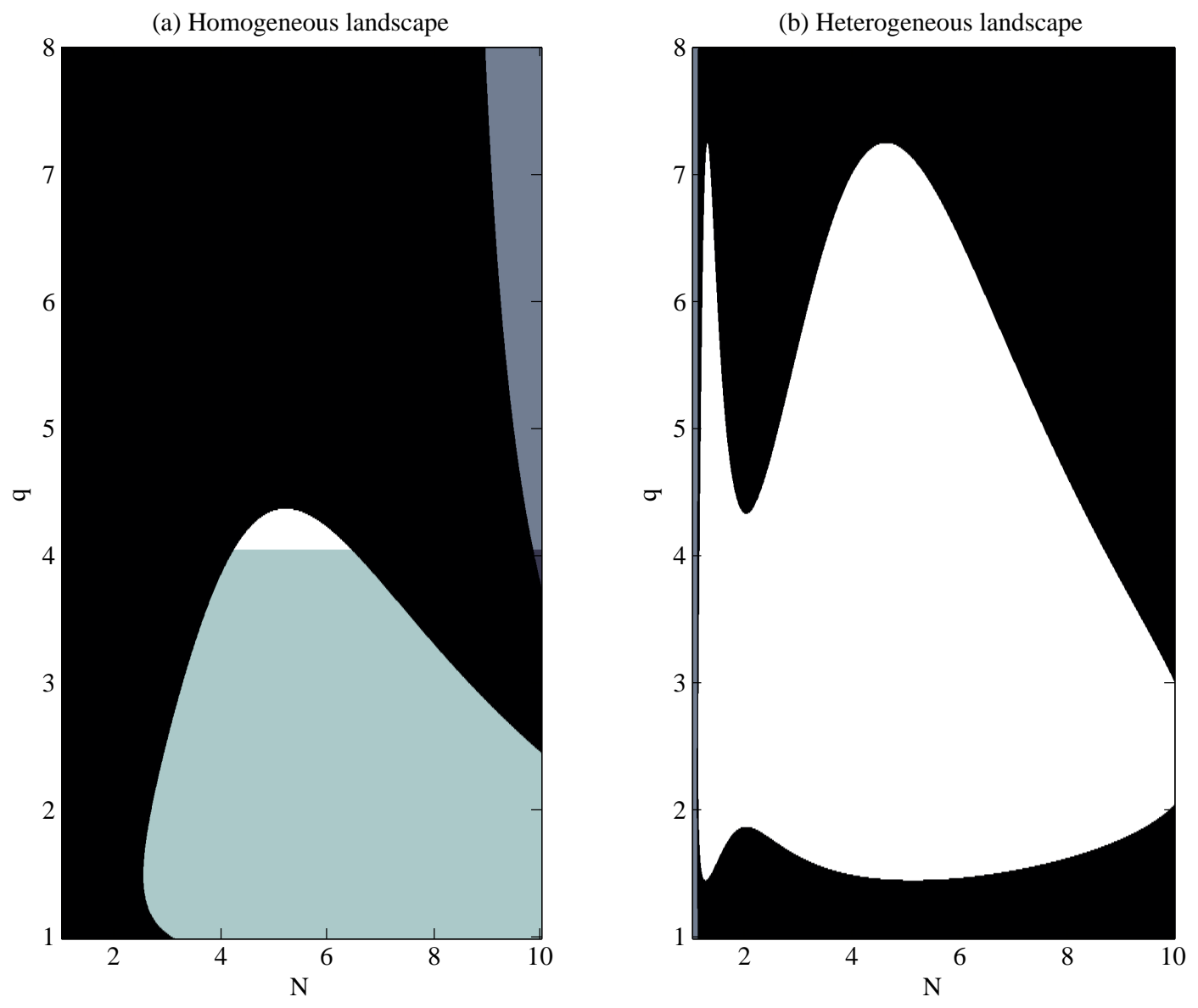

Figure 5: Comparison of stability conditions, according to the dispersion relation, between the homogeneous (panel a) and heterogeneous (panel b) landscape. The homogeneous landscape consists of only good patches whereas the heterogeneous landscape has good and bad patches alternating. White, black and dark grey colours indicate Turing instability and stability, respectively, as in previous figures. The light grey shaded region in (a) indicates that the patchindependent (period 1) solution is unstable to spatially homogeneous perturbations giving rise to population cycles and preventing Turing pattern formation. We use the baseline parameters with the exception of $\mu_{v}=10$. 
entirely.

\section{Discussion}

One of the great challenges in ecology is to explain the mechanisms behind the observed spatiotemporal variation in species densities. Such spatial variation could be (i) externally imposed in a heterogeneous landscape by variations in habitat quality, or (ii) arise on homogeneous landscapes from species interaction and dispersal through diffusion-driven instabilities or other feedback mechanisms that lead to self-organised population patterns. The former view is reflected in habitat suitability models where population abundance is correlated with local habitat features and resource availability (Ergon et al., 2001). Documenting the latter has been a highly active area of ecological research in recent years (Rietkerk et al., 2004). Examples can be found in arid ecosystems (Rietkerk and van de Koppel, 2008), marine systems (Wang et al., 2010a), and also in other areas of the biophysical sciences such as developmental biology and coupled chemical reactors (Gilbert, 1994; Horsthemke and Moore, 2004). In reality, both aspects are likely to interact (Schmitz, 2010). The strength of this interaction and the expected resulting patterns depend on the relative length scales of the different mechanisms (Sheffer et al., 2013; Benson et al., 1993b). If the spatial extent of landscape features is much larger than the length scale on which biological feedbacks (through dispersal and species dynamics) operate, then any patterns in species abundance are likely to be self-organised. If the two scales are comparable then we expect the two mechanisms to interact such that spatial patterns are more difficult to predict. Sheffer et al. (2013) propose a conceptual framework and empirical setting to explore this influence of spatial scales.

We developed a theoretical framework to understand the spatial patterns that arise in a predator-prey system where external factors and self-organisation interact. We represented the heterogeneous landscape generated by abiotic factors as a series of periodically alternating patches, and the population dynamics on each patch as a system of differential equations. While pattern formation has been studied in other contexts on homogeneous lattices and networks of patches (Wearing et al., 2000; Lubensky et al., 2011; Formosa-Jordan et al., 2012), to the best of our knowledge, our model is the first application of these ideas to spatial ecology and the first attempt to deal with strong heterogeneity (but see Webb and Owen (2004a) for a related idea). Due to the spatial heterogeneity, this system does not have a spatially constant steady state on the level of patches. Instead, there is a steady state that is spatially constant on the level of tiles. Within each tile, species densities vary between the good and bad patch, reflecting the populations tracking externally imposed landscape heterogeneity. We employed linear dispersion relation and numerical bifurcation analysis to study the stability of this steady state to spatially non-uniform perturbations as well as the occurrence of stable, spatially non-uniform (on the level of tiles) states.

We found that (i) the homogeneous (tile-level) state can be destabilised by non-constant spatial perturbations (e.g. Figure 1); that (ii) there are potentially many stable, coexisting, spatially-structured states with reasonably large basins of attraction (e.g. Figure 2). Similar results are known on homogeneous networks (Wolfrum, 2012). In addition, we find that (iii) externally imposed spatial heterogeneity seems to have the potential to promote self-organised spatial patterns (e.g. Figure 5). Sheffer et al. (2013) had reached a similar conclusion from their conceptual model of vegetation patterning. The patterns we find can be explained with the classical mechanisms (Segel and Jackson, 1972; Gierer and Meinhardt, 1972) of long-range inhibition 
(a)

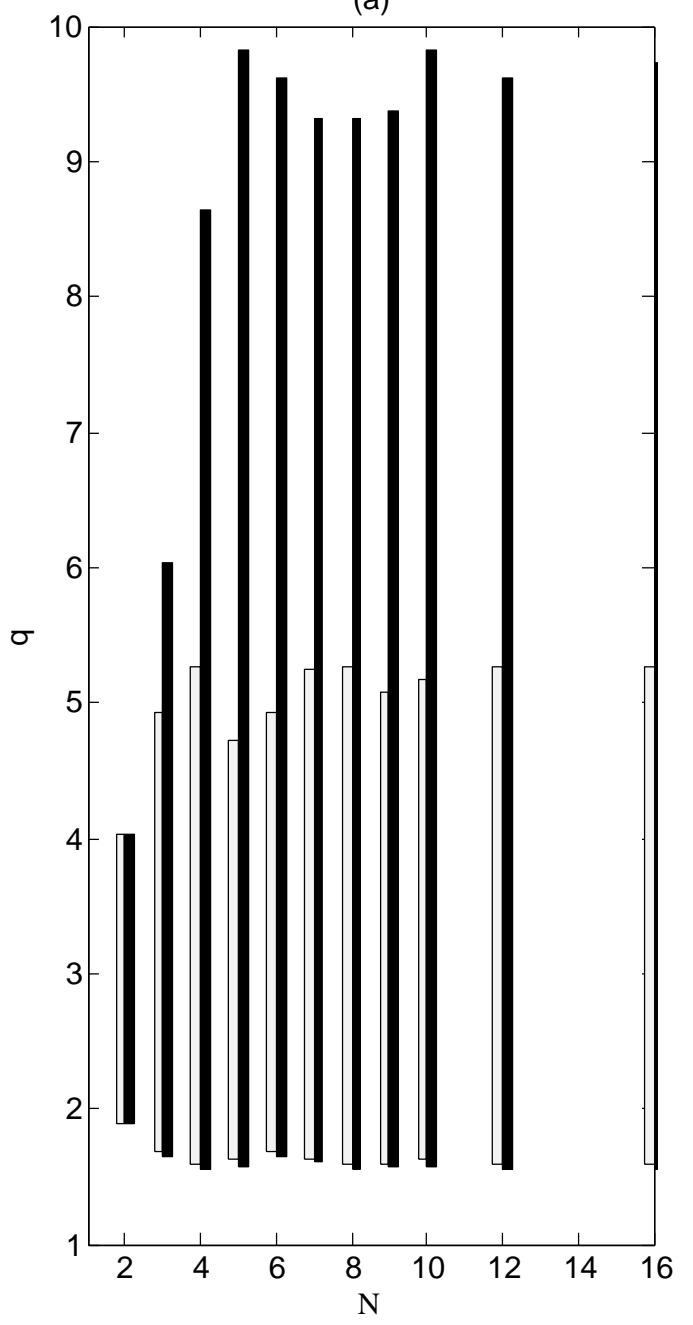

(b)

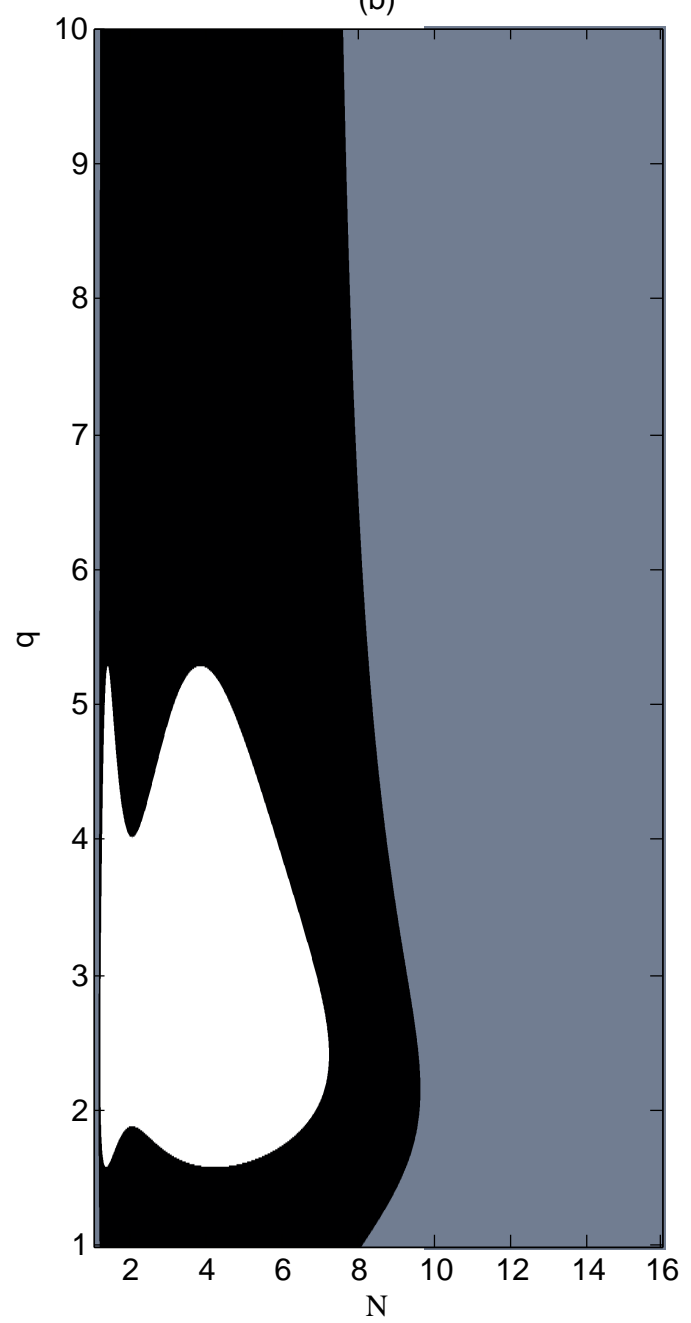

Figure 6: Stability boundary plots comparing the results from the full non-linear bifurcation analysis (a) to the results of the linear stability analysis of the patch-independent (period 1) solutions on an infinite one dimensional spatial domain (b). In (a) the hashed bars indicate the range of $q$ which give unstable patch-independent (period 1) solutions found from the numerical bifurcation analysis of the full non-linear model. The white bars indicate the full range of $q$ where patterns arise in the full non-linear model. The white region in (b) indicates values of the parameter $q$ for which we expect to obtain a pattern of wavelength $N$. In the white region the patch-independent (period 1) solution is stable to spatially homogeneous perturbations, but is unstable to spatially varying perturbations of wavelength $N$. In the black and grey regions the period 1 solution is stable to spatially varying perturbations of wavelength $N$ and patterns are not possible according to the linear analysis. The difference between the black and grey regions is that dominant eigenvalues are real and complex respectively. 
(predator) and short-range activation (prey), when properly taking into account how dispersal rates, patch residence times and landscape configuration interact to create the length scale of biological feedbacks. The difference in predator-prey dispersal ability required for long-range inhibition and short range activation is often observed in marine systems. Marine piscivores regularly migrate across spatial scales much larger than the habitat occupied by their prey (Spencer and Collie, 1995), and so marine environment may provide a good setting for potential applications of our findings.

Our results have particular implications for the management of biological systems, for example the alteration of existing habitats and the design of reserves. For example, optimal design and spacing of systems of marine reserves is usually based on maximising the likelihood of population persistence, but once persistence is guaranteed, interaction with other populations is often not considered (but see Gouhier et al. (2010)). Between two consecutive marine reserves (good patches) lies a region of unprotected habitat (bad patch) where prey death is high due to harvesting. Our results show that long-range spatial patterns may arise in such a situation. Figure 1(b), for example, shows a period-4 pattern on a system of eight tiles where the prey density in the good patches on tiles $1,4,5$, and 8 is low, even lower than the prey density in the bad patches in tiles 2 and 6 . It might be tempting to conclude that the good patches in tiles $1,4,5$, and 8 are not successful reserves that could be removed. We simulated the system with those four patches converted to bad patches (Figure 1(c)), and we found that this local change caused by patch conversion has a global effect, elevating the prey density on all of the remaining good patches. The original pattern wavelength is typically preserved and the predator densities (not shown) are also globally affected, often showing a decrease in density. These observations apply equally well to naturally heterogeneous habitats. When patterns arise in heterogeneous landscapes, the steady-state population density need not be uniformly high on good patches; it may, in fact, be lower on some good patches than on some bad patches (Figure 1). Hence, neither are low population densities in good patches a sign of impending collapse, nor is low population density a sign for low habitat quality. Both can merely be a consequence of species interaction and spatial coupling.

There are many examples of spatially periodic habitats of the type considered in our model. One particularly rich example is semi-arid vegetation, which tends to self-organise into patterns because of the positive feedback between vegetation density and water infiltration (Rietkerk et al., 2004; Meron, 2012; Sherratt, 2015). Bonachela et al. (2015) have shown that the spatial heterogeneity created by periodic patterns of termite mounds plays an important regulatory role for the vegetation patterns that develop in this heterogeneous landscape. Most notably, they predicted that the heterogeneity increases resilience to reductions in rainfall, a result in keeping with the work of Yizhaq et al. (2014) on spatial heterogeneity in soil water diffusion. Moreover the vegetation itself provides a spatially patterned habitat for other fauna, although this is an aspect of semi-arid ecosystems that has received little attention in the literature. Other examples of spatial patterns at the whole ecosystem scale include mussel beds (Wang et al., 2010a), intertidal mudflats (Weerman et al., 2012), ribbon forests (Bekker et al., 2009) and peat bogs (Eppinga et al., 2009). In each case these systems provide patterned habitats for other components of the ecosystem, although again this has been little studied, with the research focus being on the landscape patterning itself.

We have demonstrated that unless the number of tiles is small, there can be a large number of coexisting stable spatial patterns, many of which have an appreciable basin of attraction. Many of these solutions are very similar to one another, implying that populations can be in any of a range of stable patterns, which differ only slightly. For example, a variety of different patterns 
could become established on similar landscapes, depending on initial conditions or environmental perturbations. Empirical evidence for this statement comes from Sheffer et al. (2013). In the same vein, landscape alterations may have a number of unexpected consequences for population densities. Species abundances could change far beyond the range of the actual alteration if the system is moved between basins of attraction for two distinct patterns. Based on our observation that landscape heterogeneity can promote spatial patterns, landscape alterations that increase heterogeneity could lead to emergent patterns where there were none to begin with. The mechanisms that we uncovered complement those found by Page et al. (2003) in a developmental context. In Page et al.'s work, a spatial discontinuity in the population dynamic parameters drove the pattern formation, and the resulting patterns were centred around this discontinuity. In our case, the patterns are not originating from such parameter discontinuities, instead they occur across the entire domain driven by spatial coupling as well as the short-range destabilising effect of the prey and the long-range stabilising effect of the predators.

It is well known that standard Lotka-Volterra or Rosenzweig-MacArthur predator-prey models do not support diffusion-driven instabilities on homogeneous landscapes (Okubo and S.A., 2001), while the model by Leslie and May that we considered does (Mukhopadhyay and Bhattacharyya, 2006). Fasani and Rinaldi (2011) showed that the Rosenzweig-MacArthur model can readily show the required activator-inhibitor structure by including one of at least nine potential demographic factors for the predator. While not all factors enhanced the propensity for pattern formation, their result suggests that the ideas presented here may have wide applicability. Furthermore, since we observed pattern formation in the heterogeneous landscape for a much wider range of parameters than for a homogeneous landscape, and especially for parameter values where the model on an isolated good patch has oscillatory dynamics, we conjecture that most of our results are fairly robust and apply to more general predator-prey models. Some support for this conjecture comes from work by Strohm and Tyson (2009) who compared the dynamics of several predator-prey models on a simple fragmented landscape and found that results were largely insensitive to model type. Future work will have to explore how robust our results are with respect to other modelling assumptions, for example, the arrangement and sizes of patches.

Managed ecological settings are not the only context within which our work is applicable. Heterogeneous environments are also present in developmental biology. As an embryo grows, patterns are laid down in a hierarchical fashion with new patterns forming on top of earlier patterns. Spatially discrete models have been used to describe developmental pattern formation before, but not in the context of a heterogeneous domain. Instead, coupled ODEs have been used to describe juxtacrine signalling (a means of nearest neighbour communication that occurs in closely packed cells), but the assumption has been one of a homogenous spatial environment on which fine-grained patterns form in developing tissue. Our approach offers a new way to study pattern formation on a heterogenous domain. Previous studies of pattern formation had largely been limited to simple cases of spatially-dependent step-functions in diffusion or kinetic parameters (Benson et al., 1993b; Page et al., 2003).

\section{Acknowledgments}

We would like to thank the Mathematical Biosciences Institute (Ohio) who supported attendance of all three authors at the 2011 Workshop 3 on "Ecology and control of invasive species, including insects," where initial discussion of these ideas took place. Following the initial discussions, this work got underway when FL was visiting the University of Glasgow and Heriot-Watt University. This visit was supported by the London Mathematical Society and the Edinburgh Mathematical 
514 Society. FL gratefully acknowledges a discovery grant by the National Science and Engineering 515 Research Council of Canada. 


\section{Appendix A}

In section 3.1 we presented the results of a numerical bifurcation analysis of our model equations. In this Appendix we discuss the details of this method, highlighting the various technical difficulties that we encountered and how we overcame them. Readers considering reproducing the figures should be aware that they require large amounts of computer time. Taken together, all of the numerical continuations for $N=16$ took about 2 weeks on a Linux PC with a $2.83 \mathrm{GHz}$ Intel Core 2 Quad Q9500 processor.

Our basic approach is to calculate numerically the patch-independent solution for a relatively small value of $q$, and then numerically continue the solution in $q$ from this starting point, detecting bifurcations and following bifurcating branches. We performed our calculations using the software package AUTo97 (Doedel, 1981; Doedel et al., 1991, 2006). The values of key AUTO parameters are: $i p s=1$ (stationary solutions of ODEs); isp=1 (enable detection of bifurcation points); isw=1 (enable branch switching); iid=0 (minimal diagnostics; otherwise the file fort. 9 becomes extremely big). With these settings, AUTO attempts to calculate not only the primary solution branch, but also the bifurcating branches from the first $|\mathrm{mxbf}|$ bifurcation points. In principle therefore, AUTO should automatically calculate the entire bifurcation diagram in a single run. However a major difficulty arises in practice when solution branches are loops. Then the numerical continuation will typically trace round the loop several times before ending when the number of continuation steps reaches its pre-assigned maximum $\mathrm{nmx}$. Therefore each bifurcation point on the loop is recorded several times, and each occurrence acts as a starting point for a new branch calculation, causing bifurcation points along the branch to be located several times. These bifurcating branches may themselves be loops, in which case there will be multiple recording of bifurcation points for each replicate of the branch. Repetition of this process gives the potential for an exponential increase in the number of times a solution branch is calculated as a function of the number of bifurcations separating it from the primary solution branch. It turns out that looped solution branches are quite common for our equations. Moreover the same problem can occur when the numerical continuation turns around at the end of a solution branch and recomputes it in the opposite direction; in theory this should be prevented by setting $\mathrm{mxbf}<0$, but in practice it sometimes happens anyway.

This multiple calculation of bifurcation points and solution branches is a feature of all our computations. It means that however large $|\mathrm{mxbf}|$ is, the calculation will always continue until this upper limit on the number of solution branches is attained, and one can never be certain whether or not the resulting bifurcation diagram is complete. We took mxbf $=-4000$, which compares with the value $|\mathrm{mxbf}|=10$ used in most examples in the AUTO manual. The vast majority of the 4000 solution branches that are then calculated are repeats: nevertheless there may be omissions. Therefore we augmented the basic calculation with an additional step. For each value of $q$ in the set $1.0,1.25,1.51 .75, \ldots$ we ran 1000 simulations of the model equations with initial conditions in which each variable was chosen randomly from a uniform distribution between $20 \%$ and $200 \%$ of its value in the patch-independent solution. Many of the patterns generated by these simulations lie on solution branches that have already been calculated, but typically some do not, due to the incompleteness of the preliminary bifurcation diagram. In such cases, we performed separate runs of AUTO starting from the pattern found via simulation, with mxbf reset to 10; we deliberately set $m x b f>0$ in this case. During such a run, one wants to record $u_{1}^{j}$ for all values of $j$ since these should all be plotted on the bifurcation diagram; however AUTO only records up to 6 variable values (in the fort. 7 output file), which presents a problem for $N>6$. One possible remedy would be to edit the AUTO source code to output more variable values. However we adopted the alternative strategy of doing $N$ separate runs of AUTO, starting 
from $\left(u_{1}^{j}, u_{2}^{j}, v_{1}^{j}, v_{2}^{j}\right)=\left(\tilde{u}_{1}^{j+k(\bmod N)}, \tilde{u}_{2}^{j+k(\bmod N)}, \tilde{v}_{1}^{j+k(\bmod N)}, \tilde{v}_{2}^{j+k(\bmod N)}\right)$ where $\left(\tilde{u}_{1}^{j}, \tilde{u}_{2}^{j}, \tilde{v}_{1}^{j}, \tilde{v}_{2}^{j}\right)$ is the pattern found via simulation, and $k=0,1, \ldots, N-1$.

It is important to note one consequence of our two-step method for calculating the bifurcation diagrams, which is that we cannot guarantee that we have calculated all of the solution branches. Indeed, for the very complicated diagrams for $N=12$ and $N=16$, we think that it is very likely that our results omit some unstable solution branches, although given the dense network of such branches it would probably be difficult to distinguish the results visually if some additional branches were included. Because we use the results from a large volume of simulations to give starting points for numerical continuation, we think it likely that we have calculated the vast majority of the branches with stable parts. However we cannot rule out the possibility of additional stable portions of solution branches that have either very small extent in $q$, or a very small basin of attraction.

We also mention two other more minor technical difficulties, for the benefit of readers considering using our approach themselves. Firstly, in some cases AUTO erroneously detects some Hopf bifurcation points. These occur when two real eigenvalues change sign simultaneously: numerical discretisation introduces very small imaginary parts to these eigenvalues, causing AUTO to detect a Hopf bifurcation. These do not cause any difficulties in practice, and so can safely be ignored - in particular AUTO does not automatically attempt to trace limit cycle branches emanating from Hopf bifurcation points. Alternatively the "Hopf bifurcations" can be eliminated by reducing the error tolerances and step sizes. We have not found any genuine Hopf bifurcations in any of the bifurcation diagrams we calculated. Secondly, the fact that most solution branches are calculated many times causes very long rendering times for plots. To avoid this, we processed the data files before plotting, removing repeated solution branches. Specifically, we removed branches whose first 20 points were within a small tolerance of the first 20 points of a previous branch.

We end this Appendix with a full listing of the various AUTO parameters that we used in our calculations. $\mathrm{NDIM}=4 N$, IPS $=1, \mathrm{IRS}=0, \mathrm{ILP}=0, \mathrm{NICP}=1, \mathrm{ICP}=1, \mathrm{NTST}=50, \mathrm{NCOL}=4$, $\mathrm{IAD}=3, \mathrm{ISP}=1, \mathrm{ISW}=1, \mathrm{IPLT}=0, \mathrm{NBC}=0, \mathrm{NINT}=0, \mathrm{NMX}=4000, \mathrm{RL} 0=0.6, \mathrm{RL} 1=15.0, \mathrm{~A} 0=0, \mathrm{~A} 1=100$, $\mathrm{NPR}=4000, \mathrm{MXBF}=-4000, \mathrm{IID}=2, \mathrm{ITMX}=8, \mathrm{ITNW}=5, \mathrm{NWTN}=3, \mathrm{JAC}=0, \mathrm{EPSL}=10^{-7}, \mathrm{EPS}=10^{-7}$, $\mathrm{EPSS}=10^{-5}, \mathrm{DS}=0.0005, \mathrm{DSMIN}=0.0001, \mathrm{DSMAX}=0.005, \mathrm{IADS}=1, \mathrm{NTHL}=1, \mathrm{I}=11, \mathrm{THL}=0, \mathrm{NTHU}=0$, $\mathrm{NUZR}=0$. The only variation in these values was to MXBF, which was set to 10 or 1 in some runs, as discussed above.

\section{References}

M. F. Bekker, J. T. Clark, and M. W. Jackson. Landscape metrics indicate differences in patterns and dominant controls of ribbon forests in the rocky mountains, usa. Appl. Veg. Sci., 12:237-249, 2009.

D.L. Benson, P.K. Maini, and J.A. Sherratt. Analysis of pattern formation in reaction diffusion models with spatially inhomogeneous coefficicients. Math. Comput. Modelling, 17(2):29-34, 1993a.

D.L. Benson, J.A. Sherratt, and P.K. Maini. Diffusion driven instability in an inhomogeneous domain. Bull. Math. Biol., 55(2):365-384, 1993b. 
D.L. Benson, P.K. Maini, and J.A. Sherratt. Unravelling the Turing bifurcation using spatially varying diffusion. J. Math. Biol., 37:318-417, 1998.

J.A. Bonachela, R.M. Pringle, E. Sheffer, T.C. Coverdale, J.A. Guyton, K.K. Caylor, S.A. Levin, and C.E. Tarnita. Termite mounds can increase the robustness of dryland ecosystems to climatic change. Science, 347:651-655, 2015.

L. Botsford, A. Hastings, and S.D. Gaines. Dependence of sustainability on the configuration of marine reserves and larval dispersal distance. Ecology Letters, 4(144-150), 2001.

R. Cantrell, C. Cosner, and W. Fagan. The implications of model formulation when transitioning from spatial to landscape ecology. Math. Biosci., 9(1):27—60, 2012.

V. Deblauwe, N. Barbier, P. Couteron, O. Lejeune, and J. Bogaert. The global biogeography of semi-arid periodic vegetation. Global Ecol. Biogoegr., 17:715-723, 2008.

E.J. Doedel. Auto, a program for the automatic bifurcation analysis of autonomous systems. Cong. Numer., 30:265-384, 1981.

E.J. Doedel, H.B. Keller, and J.P. Kernévez. Numerical analysis and control of bifurcation problems: (i) bifurcation in finite dimensions. Int. J. Bifurcation Chaos, 1:493-520, 1991.

E.J. Doedel, W. Govaerts, Y. Kuznetsov, and A. Dhooge. Numerical continuation of branch points of equilibria and periodic orbits. In E.J. Doedel, G. Domokos, and I.G. Kevrekidis, editors, Modelling and computations in dynamical systems, pages 145-164. World Scientific, 2006.

M. B. Eppinga, P. C. De Ruiter, M. J. Wassen, and M. Rietkerk. Nutrients and hydrology indicate the driving mechanisms of peatland surface patterning. Am. Nat., 173:803-818, 2009.

T. Ergon, X. Lambin, and N. C. Stenseth. Life-history traits of voles in a fluctuating popualtion repsond to the immediate environment. Nature, 411:1043-1045, 2001.

S. Fasani and S. Rinaldi. Factors promoting or inhibiting turing instability in spatially extended prey-predator systems. Ecol. Modeling, 222:3449-3452, 2011.

P. Formosa-Jordan, M. Ibanes, S. Ares, and J.M. Frade. Regulation of neuronal differentiation at the neurogenic wavefront. Development, 139:2321-2329, 2012.

D.A. Garzón-Alvarado, C. Galeano, and J. Mantilla. Numerical tests on pattern formation in 2D heterogeneous mediums: An approach using the Schnakenberg model. Dyna, 79(172): 56-66, 2012.

A. Gasull, R. E. Kooij, and J. Torregrosa. Limit cycles in the Holling-Tanner model. Publicacions Matematiques, 41:149-167, 1997.

A. Gierer and H. Meinhardt. A theory of biological pattern formation. Kybernetik, 12:30-39, 1972.

S.F. Gilbert. Developmental biology. Sinauer, 4th edition, 1994. 
T.C. Gouhier, F. Guichard, and B.A. Menge. Ecological processes can synchronise marine population dynamics over continental scales. PNAS, 107(18):8281-8286, 2010.

W. Horsthemke and P.K. Moore. Turing instability in inhomogeneous arrays of diffusively coupled reactors. J. Phys. Chem. A, 108:2225-2231, 2004.

G.A. Jones and K.E. Sieving. Intercroppping sunflower in organic vegetab;es to augment bird predators of arthropods. Agriculture, Ecosys. and Env., 117:171-177, 2006.

S.J. Leroux, M. Larrivee, and V. Boucher-Lalonde. Mechanistic models for the spatial spread of species under climate change. Ecological Applications., 23(4):815-828, 2013.

Q.-X. Liu, A. Doelman, V. Rottschäfer, M. de Jager, P. M. Herman, M. Rietkerk, and J. van de Koppel. Phase separation explains a new class of self-organized spatial patterns in ecological systems. PNAS, 110.29:11905-11910, 2013.

D.K. Lubensky, M.W. Pennington, B.I. Shraiman, and N.E. Baker. A dynamical model of ommatidial crystal formation. PNAS, 108(27):11145-11150, 2011.

R. May. Stability and complexity in model ecosytems. Princeton University Press, 1974.

E. Meron. Pattern-formation approach to modelling spatially extended ecosystems. Ecol. Modelling, 234:70-82, 2012.

B. Mukhopadhyay and R. Bhattacharyya. Modeling the role of diffusion coefficients on turing instability in a reaction-diffusion predator-prey system. Bull. Math. Biol., 68:293-313, 2006.

J. D. Murray. Mathematical Biology I: An Introduction. Springer-Verlag, Berlin, 2001.

R.D. O'Dea and J.R. King. Multiscape analysis of pattern formation via intercellular signalling. Math. Biosci., 231:172-185, 2011.

R.D. O'Dea and J.R. King. The isolation of spatial patterning modes in a mathematical model of juxtacrine cell signalling. Math. Med. Biol., 30(2):95-113, 2013.

A. Okubo and Levin S.A. Diffusion and ecological problems: Modern perspective. SpringerVerlag, second edition, 2001.

M. Owen and J.A. Sherratt. Mathematical modelling of juxtacrine cell signalling. Math. Biosci., 153:125-150, 1998.

M. Owen, J.A. Sherratt, and H.J. Wearing. Lateral induction by juxtacrine signaling is a new mechanism for pattern formation. Developmental Biology, 217:54-61, 2000.

K. Page, P.K. Maini, and N.A.M. Monk. Pattern formation in spatially heterogeneous Turing reaction-diffusion models. Physica D, 181:80-101, 2003.

K. Page, P.K. Maini, and N.A.M. Monk. Complex pattern formation in reaction-diffusion systems with spatially varying parameters. Physica D, 202:95-115, 2005.

A.J. Perumpanani, J.A. Sherratt, and P.K. Maini. Phase differences in reaction-diffusionadvection systems and applications to morphognesis. IMA J. Appl. Math., 55:19-33, 1995. 
S. Petrovskii and H. Malchow. Wave of chaos: new mechanism of pattern formation in spatiotemporal population dynamics. Theor. Pop. Biol., 59:157-174, 2001.

M. Rietkerk and J. van de Koppel. Regular pattern formation in real ecosystems. Trends Ecol. Evol., 23(3):169-175, 2008.

M. Rietkerk, M.C. Boerlijst, F. van Langevelde, R. HilleRisLambers, J. van de Koppel, H.H.T. Prins, and A. de Roos. Self-organisation of vegetation in arid ecosystems. Am. Nat., 160: 524-530, 2002.

M.G. Rietkerk, S.C. Dekker, P.C. Ruiter, and J. van de Koppel. Self-organized patchiness and catastrophic shifts in ecosystems self-organized patchiness and catastrophic shifts in ecosystems self-organized patchiness and catastrophic shifts in ecosystems. Science, 305:1926-1929, 2004.

K. Sato and Y. Iwasa. Modeling of wave regeneration in subalpine abies forests: population dynamics with spatial structure. Ecology, 74:1538-1550, 1993.

O.J. Schmitz. Spatial dynamics and ecosystem functioning. PLOS Biol., 8:e1000378, 2010.

L.A. Segel and J.L. Jackson. Dissipative structure: an explanation and an ecological example. J. Theor. Biol., 37:545-559, 1972.

E. Sheffer, J. von Hardenberg, H. Yizhaq, M. Shachak, and E. Meron. Emerged or imposed: a theory on the role of physical templates and self-organisation for vegetation patchiness. Ecol. Lett., 127-139:16, 2013.

J.A. Sherratt. History-dependent patterns of whole ecosystems. Ecological Complexity, 14:8-20, 2013.

J.A. Sherratt. Using wavelength and slope to infer the historical origin of semi-arid vegetation bands. PNAS, 112:4202-4207, 2015.

J.A. Sherratt, M.A. Lewis, and A.C. Fowler. Ecological chaos in the wake of invasion. PNAS, 92:2524-2528, 1995.

J.A. Sherratt, X. Lambin, C.J. Thomas, and T.N. Sherratt. Generation of periodic waves by landscape features in cyclic predator-prey systems. Proc. R. Soc. Lond. B, 269:327-334, 2002.

E. Siero, A. Doelman, M.B. Eppinga, J.D.M. Rademacher, M. Rietkerk, and Siteur K. Striped pattern selection by advective reaction-diffusion systems: resilience of banded vegetation on slopes. Chaos, 25:036411, 2015.

K. Siteur, E. Siero, M.B. Eppinga, J.D.M. Rademacher, A. Doelman, and M. Rietkerk. Beyond turing: the response of patterned ecosystems to environmental change. Ecological Complexity, 20:81-96, 2014.

P. D. Spencer and J. S. Collie. A simple predator-prey model of exploited marine fish populations incorporating alternative prey. ICES J. Marine Sci., 53:615-628, 1995.

S. Strohm and R. Tyson. The effect of habitat fragmentation on cyclic population dynamics: a numerical study. Bull. Math. Biol., 71:1323-1348, 2009. 
P. Turchin. Complex Population Dynamics. Princeton University Press, Princeton, 2001.

S. van der Stelt, A. Doelman, G. Hek, and J.D.M. Rademacher. Rise and fall of periodic patterns for a generalized Klausmeier-Gray-Scott model. J. Nonlinear Sci., 23:39-95, 2013.

J.-P. Voroney, A.T. Lawniczak, and R. Kapral. Turing pattern formation in heterogeneous media. Physica D, 99:303-317, 1996.

R.H. Wang, Q.X. Liu, G.Q. Sun, Z. Jin, and J. Van de Koppel. Nonlinear dynamic and pattern bifurcations in a model for spatial patterns in young mussel beds. J. R. Soc. Interface, 6(37): 705-718, 2010a.

W.M. Wang, L. Zhang, H.L. Wang, and Z.Q. Li. Pattern formation of a predator-prey system with Ivlev-type functional response. Ecol. Modeling, 221:131-140, 2010b.

H.J. Wearing and J.A. Sherratt. Nonlinear analysis of juxtacrine patterns. SIAM J. Appl. Math, 62:283-309, 2001.

H.J. Wearing, M. Owen, and J.A. Sherratt. Mathematical modelling of juxtacrine patterning. Bull. Math. Biol., 62:293-320, 2000.

S.D. Webb and M. Owen. Intra-membrane ligand diffusion and cell shape modulate juxtacrine patterning. J. Theor. Biol., 230:99-117, 2004a.

S.D. Webb and M. Owen. Oscillations and patterns in spatially discrete models for developmental intercellular signalling. J. Math. Biol., 48(4):444-476, 2004b.

E.J. Weerman, J. Van Belzen, M. Rietkerk, S. Temmerman, S. Kefi, P.M.J. Herman, and J. Van de Koppel. Changes in diatom patch-size distribution and degradation in a spatially self-organized intertidal mudflat ecosystem. Ecology, 93:608-618, 2012.

M. Wolfrum. The turing bifurcation in network systems: Collective patterns and single differentiated nodes. Physica D, 241:1351-1357, 2012.

H. Yizhaq, S. Sela, T. Svoray, S. Assouline, and G. Bel. Effects of heterogeneous soil-water diffusivity on vegetation pattern formation. Water Resour. Res., 50:5743-5758, 2014.

Y.R. Zelnik, E. Meron, and G. Bel. Gradual regime shifts in fairy circles. PNAS, page doi: $10.1073 /$ pnas. 1504289112 , in press. 